\title{
Preface of the "Symposium on Interactive Computational Modelling in Science, Technology, Engineering and Mathematics Education"
}

Cite as: AIP Conference Proceedings 2293, 400001 (2020); https://doi.org/10.1063/5.0026569 Published Online: 25 November 2020

\section{Rui Gomes Neves}

\section{ARTICLES YOU MAY BE INTERESTED IN}

Interactive computational modelling to improve teaching of physics and mathematics in marine geophysics

AIP Conference Proceedings 2293, 400003 (2020); https://doi.org/10.1063/5.0026601

Physics education with interactive computational modelling AIP Conference Proceedings 2293, 400002 (2020); https://doi.org/10.1063/5.0026566

The maximum number of periodic solutions for a deployable circular mesh antenna model AIP Conference Proceedings 2293, 390005 (2020); https://doi.org/10.1063/5.0026767

Challenge us. What are your needs for periodic signal detection?

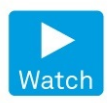

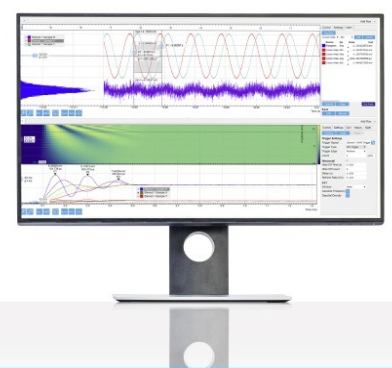

Zurich - Instruments 


\title{
Preface of the "Symposium on Interactive Computational Modelling in Science, Technology, Engineering and Mathematics Education"
}

\author{
Rui Gomes Neves ${ }^{1,2, a), b)}$ \\ ${ }^{1}$ Departamento de Ciências Económicas e Empresariais (DCEE), Universidade Autónoma de Lisboa (UAL), \\ Portugal \\ ${ }^{2}$ Unidade de Investigação Educação e Desenvolvimento (UIED), CICS.NOVA - Centro Interdisciplinar de \\ Ciências Sociais, Faculdade de Ciências e Tecnologia, Universidade Nova de Lisboa (FCT/UNL), Portugal \\ a)rneves@autonoma.pt \\ b) rgn@fct.unl.pt
}

The structures of knowledge of science, technology, engineering and mathematics (STEM) are developed in modelling processes involving epistemologies and cognition frames that balance different elements from theory, experimentation and scientific computation. Increasingly, many such processes appeal to advanced mathematical physics models and methods scientific computation. As a consequence, STEM educational curricula, methodologies and teaching-learning environments should be based on strategies able to ensure the development of technological and computationally enriched meaningful learning paths, that go through balanced interactive explorations of all the different phases of the modelling cycle, namely, qualitative contextual description, definition, exploration, interpretation and validation of mathematical models, communication of results and generalizations. Considering specific adaptations for specific area dependent contexts, such paths should bring up strong interconnected backgrounds in physics, mathematics and scientific computation while achieving as early as possible a balanced integration of computational modelling. Indeed, in contemporary STEM activities computational modelling, understood as modelling with computational knowledge, cognition and technologies, plays a fundamental role in the expansion of knowledge and cognitive horizons as it generates enhanced calculation, exploration, visualization and validation capabilities for the creation of models able to represent underlying STEM structures or mechanisms. Likewise, the expectation is that STEM learning processes can effectively become more meaningful with an appropriate integration of computational modelling.

Over the years many educational research results have been gathered in support of this expectation, showing that computers can be used as cognitive artifacts able to improve fundamental familiarization and reification processes associated with learning in various STEM contexts. Indeed, as cognitive instruments computers have been shown to allow the creation of interactive learning environments in which abstract STEM conceptual entities can be perceived as concrete-abstract objects. Concrete because they can be manipulated on the screen and react as real objects, and abstract because they can be scientific or mathematical constructs. Such real-time on-screen interaction with STEM constructs enhances learning cognitive activities, leading to improved familiarization and reification, and consequently more meaningful learning. Computers also allow the introduction of modelling with numerical analysis in the learning processes, complementing analytical studies. Multiple representations of mathematical models, e.g. graphs, tables and simulations, can be explored and the interconnection between the analytical and numerical approaches discussed. In many instances, numerical methods turn out to be conceptually simpler than the analytical methods and the focus can be on meaning and semi-quantitative reasoning. Moreover, the exploration of more realistic problems is possible at an earlier learning stage when the level of knowledge and cognition required by the relevant analytical methods has not yet been reached.

However, most current practices in STEM education courses remain oblivious to the increasing amount of available scientific results from the sciences of education and are still not capable of fully reflecting STEM epistemological and cognitive characteristics. Computers, for instance, are largely used as passive objects for the simple display of text, images or simulations, and not as true cognitive artifacts for modelling integrated in interactive engagement environments appropriately approximating contemporary STEM research environments. This problem is present in all educational levels and is a relevant factor contributing for the existence of many students with fragmented knowledge states, deteriorating expectations and often low exam success rates. There are many reasons behind this problem. On one hand, it is clear that educational research results and innovative didactic

International Conference of Numerical Analysis and Applied Mathematics ICNAAM 2019

AIP Conf. Proc. 2293, 400001-1-400001-2; https://doi.org/10.1063/5.0026569

Published by AIP Publishing. 978-0-7354-4025-8/\$30.00 
practices can only have widespread dissemination and implementation in an open democratic society sustaining appropriate levels of investment in people and infrastructures, and that the associated developments are incompatible with narrow and shortsighted visions imposing excessive austerity policies. Indeed, with such kind of policies it is not just a temporary shortage of material or financial resources that is at stake. Feedback effects across society tend to favor closed and corrupt authoritarian structures that prevent the creation of the necessary conditions for innovation and for the implementation of innovation, thus leading to more persistent civilizational regressions that are subsequently much more difficult to revert. On the other hand, it is also clear that there are numerous scientific and didactic challenges related to this problem whose complete understanding and resolution remains open.

For example, in many STEM educational contexts research has shown that the learning processes can be improved when students work on problem-based activities in computer enriched interactive engagement environments approximately recreating the epistemological and cognitive involvement of STEM modelling processes. In this context, a variety of computational modelling activities have demonstrated enhanced capacity and efficacy for helping in the generation of better knowledge development and structuring, and better resolution of cognitive conflicts with prior knowledge based on common sense or incorrect scientific ideas. Still, scientific knowledge about the dynamical epistemological and cognitive processes that are at play while learning the different STEM subjects, and the corresponding mapping of student's modelling paths, reasoning strategies, knowledge structures and learning difficulties, remains fragmentary. Moreover, deeper comparative analysis between research and didactic approaches is also needed. In this Symposium we present contributions that aim to improve on this situation, focusing on STEM introductory and intermediate university education and STEM secondary education.

\section{Rui Gomes Neves}

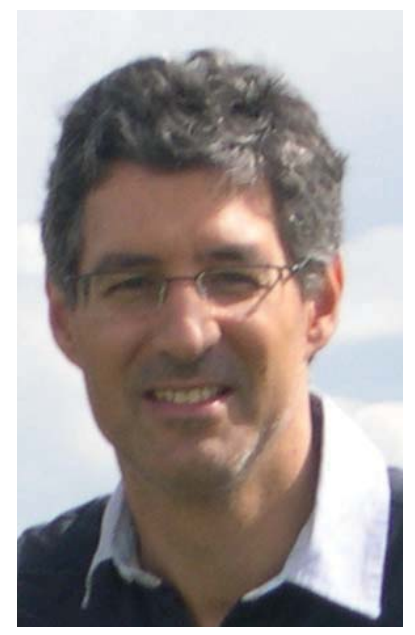

Rui Gomes Mendonça Neves studied physics first at Universidade de Lisboa, Portugal, and last at University of Durham, UK, where in 1997 he obtained his $\mathrm{PhD}$ degree in physics. After the PhD, between 1997 and 2007, Rui Neves worked as Postdoctoral Research Fellow and Assistant Professor, mainly at Universidade do Algarve, Centro Multidisciplinar de Astrofísica/Instituto Superior Técnico (CENTRA/IST) and Centro de Electrónica, Optoelectrónica e Telecomunicações (CEOT), Portugal. Between 2008 and 2015 he was Assistant Investigator at Unidade de Investigação Educação e Desenvolvimento (UIED) and Faculdade de Ciências e Tecnologia, Universidade Nova de Lisboa (FCT/UNL), Portugal, and Visiting Professor at Universidade do Vale do Taquari - Univates, Brazil. Since 2016 Rui Neves is Assistant Professor at Universidade Autónoma de Lisboa with research membership in UIED, CICS.NOVA - Centro Interdisciplinar de Ciências Sociais, FCT/UNL. Rui Neves research interests are: (1) theoretical physics and applications, focusing on high energy physics and gravitation, and on applications to biomedical sciences, geosciences and quantum computers, and (2) sciences of education, namely, knowledge and cognition development, computers, modelling and interactive environments in physics and mathematics for STEM education. 